\title{
Efficacy of Microecopharmaceutics Combined with Early Enteral Nutrition Support in the Treatment of Severe Acute Pancreatitis
}

\author{
Zhaolei Qiu, Feng Cheng, Hai Jiang, Lei Li, Chuanming Zheng, Zhaohui Du and Zhenjie Wang \\ Department of Emergency Surgery, The First Affiliated Hospital of Bengbu Medical College, Bengbu City, Anhui Province, China
}

\begin{abstract}
To explore the efficacy of microecopharmaceutics combined with early enteral nutrition support to the treatment of severe acute pancreatitis (SAP). A total of 26 patients diagnosed with SAP in our hospital were retrospectively reviewed. Microecopharmaceutics, the living microbial food supplements that are beneficial to the host by improving the balance of intestinal microorganisms, were combined with early enteral nutrition group, and used as the treatment group; and delayed enteral nutrition was used as the control group. Two weeks after both treatments, variables of C-reactive protein (CRP), albumin (Alb), total serum protein (TP), urinary amylase recovery time, hemodiastase recovery time, acute physiology and chronic health evaluation (APCHE) score, and hospitalisation time in both groups were compared. Alb and TP of the treatment group were significantly higher than those in the control group $(p<0.05)$. The APCHE score and CRP in the treatment group were significantly lower than those in the control group $(p<0.05)$. The urinary amylase recovery time, hemodiastase amylase recovery time, and hospitalisation time in the treatment group were significantly shorter than those in the control group. The use of microecopharmaceutics combined with early enteral nutrition can effectively protect the intestinal barrier function, which is an effective treatment in patients with SAP.
\end{abstract}

Key Words: Early enteral nutrition, Probiotics, Severe acute pancreatitis, Delayed enteral nutrition.

How to cite this article: Qiu Z, Cheng F, Jiang H, Li L, Zheng C, Du Z, Wang Z. Efficacy of microecopharmaceutics combined with early enteral nutrition support in the treatment of severe acute pancreatitis. $J$ Coll Physicians Surg Pak 2020; 30(1):96-98.

Severe acute pancreatitis (SAP) is difficult to treat with characteristics of acute onset, serious illness, and high fatality rate. Some studies have demonstrated that gut flora translocation is important in SAP generation and development; and the enteral nutrition treatment can provide nutrient substrate to the body, preventing disorder of cell metabolism, regulating the immunity and maintaining the structure and function of organs and body. ${ }^{1}$ But the timing of starting enteral nutrition is still under discussion. Microecopharmaceutics combined with early enteral nutrition (within 48 hours of admission) in the treatment of SAP has rarely been studied.

The aim of this study was to determine the effectiveness of microecopharmaceutics combined with early enteral nutrition in the treatment of SAP compared to delayed enteral nutrition.

A total of 26 SAP patients of our hospital from January 2013 to November 2017 were enrolled and all met the requirement of Guidance for the Diagnosis and Treatment of Acute Pancreatitis in China, for SAP which was designed by the Pancreatology Group of Chinese Society of Gastroenterology. Among all enrolled patients,

Correspondence to: Dr. Zhenjie Wang, Department of Emergency Surgery, The First Affiliated Hospital of Bengbu Medical College, Bengbu City, Anhui Province, 233000, China

E-mail: zhenjiewang_1@126.com

Received: August 27, 2018; Revised: June 17, 2019;

Accepted: June 21, 2019
13 received the microecopharmaceutics combined with early enteral nutrition (within 48 hours of admission) and formed the treatment group, another 13 patients received the delayed enteral nutrition (after 48 hours of admission), and formed the control group. In the control group, the mean age was $26.8 \pm 5.2$ years. Among these, five patients had diabetes mellitus, two patients had acute renal dysfunction and four patients, acute respiratory distress. In the treatment group, the mean age was $33.4 \pm 5.7$ years. Among them, two patients had diabetes, one patient, acute renal dysfunction and three patients, acute respiratory distress. All patients signed the informed consent. There was no statistical difference in demographic charac-teristics, with comparability $(p>0.05)$. All patients underwent the ultrasonic-guided placement of naso-enteral nutrition tube after admission.

After two weeks of treatment, Ranson score, APACHE II score, hemodiastase recovery time, urinary amylase recovery time, hospitalisation time, TP, CRP and Alb of both groups were compared. Blood and urinary amylase recovery time and hospitalisation time were assessed by the APACHE II score.

After treatment for two weeks, TP and Alb in the treatment group were significantly increased when compared with the control group $(p<0.05)$. CRP in the treatment group was significantly lower than that in the control group $(p<0.05$, Table I).

Hospitalisation time, urinary amylase recovery time, and hemodiastase recovery time of the treatment group were 
significantly shorter than those of the control group $(p<0.05$, Table II).

Before treatment, APACHE II score in two groups was not statistically significant ( $p>0.05)$; after treatment, APACHE II score in the treatment group was lower than that in the control group $(p<0.05$, Table III).

Severe gastrointestinal paralysis, edema and gastrointestinal dysfunction often occur in the early stage of SAP, and there is no effective and specific treatment. Enterogenic infection plays a leading role in SAP and can influence SAP's prognosis. SAP can be divided into two stages: (i) In the early stage, trypsin activation induces the release of inflammatory mediators, and leads to serious cascade reaction, thereby causing the systemic inflammatory response syndrome (SIRS) and multiple organ dysfunction (MOD); (ii) Because inflammatory factors and endotoxins may cause hypofunction of intestinal mucosal barrier and gut flora translocation the peri-pancreatic and systemic infection can lead to MOD. 2

SAP-induced pancreatic tissue infection and structural change, SIRS and MOD pose threats to patients' lives. Among the mortality of SAP, $80 \%$ is due to secondary infection of pancreas and peri-pancreatic tissue, among which $90 \%$ is caused by the gut flora translocation. Some studies have demonstrated that lung is the most common involved non-pancreatic organ in SAP patients. Lung damage is the main factor leading to early death of SAP patients. ${ }^{3}$

Therefore, protecting intestinal mucosal barrier function and reducing gut flora translocation and mucosal barrier function damage in preventing crucial to SAP progression to MOD and adult respiratory distress syndrome (ARDS). Many SAP patients suffer from long-term negative nitrogen

Table I: Comparisons of TP, ALB and CRP in two groups after treatment $(n=26)$.

\begin{tabular}{l|c|c|c|c}
\hline Patient groups & $\mathrm{n}$ & TP $(\mathrm{g} / \mathrm{L})$ & ALB $(\mathrm{g} / \mathrm{L})$ & $\mathrm{CRP}(\mathrm{mg} / \mathrm{L})$ \\
\hline Control & 13 & $47.6 \pm 3.8$ & $19.0 \pm 3.2$ & $110.69 \pm 14.8$ \\
Treatment & 13 & $58.3 \pm 7.7$ & $30.9 \pm 5.6$ & $47.8 \pm 12.6$ \\
p-value & & $<0.05$ & $<0.01$ & $<0.01$ \\
\hline
\end{tabular}

Table II: Comparisons of hospitalisation time, recovery time of urinary amylase, and recovery time of blood amylase in two groups $(n=26)$.

\begin{tabular}{l|c|c|c|c}
\hline Patient groups & $\mathrm{n}$ & $\begin{array}{c}\text { Hospitalization } \\
\text { time (days) } \\
\text { (days) }\end{array}$ & $\begin{array}{c}\text { Recovery time of } \\
\text { urinary amylase } \\
\text { (days) }\end{array}$ & $\begin{array}{c}\text { Recovery time of } \\
\text { blood amylase } \\
\text { (days) }\end{array}$ \\
\hline Control & 13 & $25.3 \pm 6.1$ & $17.2 \pm 32$ & $10.8 \pm 5.3$ \\
Treatment & 13 & $15.9 \pm 5.6$ & $15.8 \pm 5.2$ & $8.4 \pm 2.5$ \\
p-value & & $<0.001$ & $<0.001$ & $<0.001$ \\
\hline
\end{tabular}

Table III: Comparisons of APACHE II score between two groups before and after treatment $(n=26)$.

\begin{tabular}{l|c|c|c}
\hline Patient groups & $\mathrm{n}$ & Before treatment & After treatment \\
\hline Control & 13 & $12.4 \pm 2.9$ & $10.2 \pm 3.8$ \\
\hline Treatment & 13 & $9.8 \pm 5.7$ & $7.6 \pm 2.3$ \\
\hline p-value & & $>0.05$ & $>0.05$ \\
\hline
\end{tabular}

balance and nutritional and metabolic dis-orders, resulting in poor health, malnutrition, decreased immunity and MOD. TNF- $\alpha$ is vital in inflammatory reaction of tissues and can lead to myocardial damage. 4 In the acute reaction phase, excessive release of proinflammatory mediator (like TNF- $\alpha$ ) can directly lead to the histiocyte necrosis, while insufficient secretion of anti-inflammatory cytokine (like IL-10) can induce the SIRS. If timely nutrition and energy are not provided, serious negative nitrogen balance may occur, thereby leading to MOD and death. Therefore, nutrition support in acute phase is important in improving SAP prognosis.

Enhanced nutrition support is a vital part of individualised and comprehensive treatment in SAP. Early nutrition support, including enteral nutrition (TPN) support and total parenteral nutrition support, are crucial to SAP treatment. But the long-term TPN support may lead to over-apoptosis of epithelia, intestinal mucosal atrophy, significant decrease of DNA and protein in intestinal mucosal cells, decrease of intestinal secretory $\operatorname{lgA}$ and intestinal motility, damage to mucosal barrier functions, increase of intestinal permeability and change of gut flora. Enteral nutrition support can resist the immune stress, protect the barrier function, increase organ and intestinal mucous hemoperfusion and decrease the permeability. Enteral nutrition can significantly decrease the abdominal and respiratory infection caused by gut flora translocation, decrease the SIRS, improve the body's immunity and promote the recovery from SAP. 5 Enteral nutrition support causes little stimulation to pancreas and makes pancreas stagnate, which conforms to the "rest" theory of pancreatitis treatment and is beneficial to recovery of SAP patients. Enteral nutrition support can provide enough energy to SAP patients, adjust the flora balance and gastrointestinal function, decrease intestinal inflammatory reaction, adjust the secretion of intestinal cytokines and decrease the MOD. 6

Microecopharmaceutics refers to using the normal flora to correct the microecological disorder, maintain the microecological balance and improve the probiotics.

The timing of starting enteral nutrition is still under discussion and the microecopharmaceutics combined with early enteral nutrition support to treat the SAP is still debatable. On the basis of enteral nutrition, the probiotics are added, to control the over-growth of pathogenic bacteria, promote intestinal micro-environment, maintain the flora balance and protect the intestinal function. Results from this study showed that microecopharmaceutics combined with early enteral nutrition support can significantly increase the TP and Alb, decrease APACHE II score and CRP, as well as shorten the hospitalisation time, urinary amylase recovery time and hemodiastase recovery time. Compared with the delayed enteral nutrition treatment, the difference had statistical significance $(p<0.05)$. Microecopharmaceutics combined with early enteral nutrition support was well 
tolerated by patients. Thus, microecopharmaceutics combined with early enteral nutrition support can promote and maintain the flora balance and adjust the production of Alb and TP, reduce the serum CRP, damage to intestinal mucosal barrier function and intestinal inflammatory reaction, so as to improve APACHE II score and shorten the hospitalisation time, urinary amylase recovery time and hemodiastase recovery time, which is beneficial for the recovery of SAP patients.

To conclude, microecopharmaceutics combined with early enteral nutrition support in the treatment of SAP patients can maintain the balance of gut flora, reduce the damage to intestinal mucosal barrier function, increase body immunity and improve enteral nutrition. This treatment is safe and effective in the treatment of SAP patients.

\section{FUNDING SOURCE:}

(1) Natural Science Research Project of Anhui Higher Education Institution (KJ2015B113by).

(2) Anhui Bengbu Medical Science and Technology Development Fund Project (BYKF1768).

(3) Peking Union Medical College Emergency Medicine Fund - Ruiyi Fund (R2018004).

\section{CONFLICT OF INTEREST:}

Authors declared no conflict of interest.

\section{AUTHORS' CONTRIBUTION:}

$Z Q, F C$ : Conception and design of the work; the acquisition, analysis, or interpretation of data for the work.
HJ, LL, CZ: Acquisition, of data for the work; final approval of the manuscript.

ZD: Acquisition of data for the work; drafting the work and final approval of the version to be published.

ZW: Conception or design of the work; drafting the work and final approval of the manuscript.

\section{REFERENCES}

1. Trikudanathan $G$, Arain M, Attam R, Freeman ML. Interventions for necrotizing pancreatitis: an overview of current approaches. Expert Rev Gastroenterol Hepatol 2013; 7:463-75.

2. Zhang KY. Study of clinical efficasy and safety of combined administration of ceftri-axone sodium and anisodamine for children with acute gastroenteritis via intravenous drip. China \& Foreign Medical Treatment 2016; 31:142-4.

3. Yu J, Jin H, Chen C, Wang WX, Deng WH, Chen XY. Poly (ADP-ribose) polymerase inhibitor attenuates expression of inflammatory mediators and injury of lung in a rat model of severe acute pancreatitis. Chinese J Emerg Med 2012; 21:38-42.

4. Qiu Z, Wang Z, Cheng F, Zheng C, Li L, Jiang H, et al. The effects of TNF- $\alpha$ and anti-TNF- $\alpha$ McAb in myocardial injury of rats with traumatic hemorrhagic shock. Biomed Res 2017; 28: 5103-6.

5. Fang LL, Hashim Z, Said SM, Than LTL, Hashim JH, Dan NC. Fractional exhaled nitric oxide (FeNO) among office workers in an academic institution, Malaysia-associations with asthma, allergies and office environment. J Asthma Res 2015; 53: 170-8.

6. Sun JK, Mu XW, Li WQ, Tong ZH, Li J, Zheng SY. Effects of early enteral nutrition on immune function of severe acute pancreatitis patients. World J Gastroenterol 2013; 19:917-22. 